PHYSICAL REVIEW D 88, 081501(R) (2013)

\title{
No consistent bimetric gravity?
}

\author{
S. Deser* \\ Lauritsen Lab, Caltech, Pasadena, California 91125, USA \\ and Physics Department, Brandeis University, Waltham, Massachusetts 02454, USA \\ M. Sandora ${ }^{\dagger}$ \\ Department of Physics, University of California, Davis, California 95616, USA
}

A. Waldron ${ }^{*}$

Department of Mathematics, University of California, Davis, California 95616, USA

(Received 15 August 2013; published 8 October 2013)

\begin{abstract}
Consistency of bimetric gravity relies on its having a gauge invariant, partially massless, limit. However, just as for single metric massive gravity, we argue that no such limit exists.

DOI: 10.1103/PhysRevD.88.081501

PACS numbers: 04.20.- q, 04.50.Kd
\end{abstract}

\section{INTRODUCTION}

Two-tensor, bimetric (or " $f$-g") gravity (BMG) was born as the fusion of the strong-interaction resonances of the 1960s (specifically that of a massive spin 2 field) with general relativity's (GR) massless metric [1]. The idea was to join the two symmetrically by giving each its own Einstein-Hilbert action, then coupling the two resulting "spaces" through a nonderivative "mass term" that necessarily reduced the separate coordinate invariances to a single one, each field transforming as a tensor (and the mass term as a scalar density). It was soon realized that upon setting, by hand, one metric to be a background, BMG could be reduced to a single metric, but nongeometric, massive gravity (mGR) theory with explicit mass terms such that the linear limit yielded the free, Fierz-Pauli (FP) field [2]. These models enjoy the isometries of the background metric as symmetries (so are Lorentz invariant massive spin 2 theories in Minkowski backgrounds). However, interest was soon brought to a halt when they were found to propagate ghost modes at nonlinear level [3], even for generic mass terms which reduced linearly to FP (coupled to a massless graviton for BMG). The obstruction was the appearance of a sixth-necessarily ghostmassive "bulk" degree of freedom (d.o.f.). It was only some forty years later that this obstruction was averted [4] by the discovery of-exactly 3-mass terms for which only 5 massive d.o.f.'s remain, all nonghost (one of the preferred mass terms was actually proposed in [2] soon after [1], and had been explicitly exempted in the no-go list of [3]). This "no-ghost" result was quickly extended to BMG with the same class of mass terms [5]. These results opened an instant, now major industry—see [6] for an early review.

\footnotetext{
*deser@brandeis.edu

sandora@ms.physics.ucdavis.edu

*wally@math.ucdavis.edu
}

Philosophically, evasion of the classical mGR no-go theorem would be unsatisfactory, as it would demote GR from its (Yang-Mills-like) status as an isolated theorywithout permitted massive neighbors-whose local symmetry cannot be broken by hand. However, absence of a sixth massive, ghostlike mode is only a necessary, but not sufficient, condition for the ultimate consistency of mGR. In fact, it was rapidly realized that these improved mGR models exhibited superluminality in their auxiliary sector and decoupling limit $[7,8]$, and quite generally suffered (local) acausalities and tachyonic modes $[9,10]$ whose interactions with (hitherto) normal matter entail catastrophic consequences [11-14]. Separately, detailed analysis of mGR's cosmological properties showed it to exhibit ghost instabilities about its homogeneous solutions [15]. The one remedy that might have saved $\mathrm{mGR}$ also failed; it cannot be made partially massless (PM): recall, in background de Sitter (dS) spaces, there exist irreps of spin 2 with only 4, rather than the 5, d.o.f.'s of flat space, when the mass and $\Lambda$ are suitably tuned. Indeed, not only is the offending mode removed in PM, but the four remaining, helicity $(2,1)$ d.o.f.'s all propagate exactly on the dS light cone, further linking PM with causal behavior [16]. However, the PM limit for single metric, two derivative theories is subject to various no-go results: first, a study of PM-gauge invariant vertices gave a no-go result at quartic order [17]. An analysis of possible truncations of Weyl-squared, conformal gravity (CG) - whose spectrum consists of relatively ghost graviton and PM modes $[18,19]$ - to its PM sector also yielded a negative result [19]. Finally, two independent groups [10,20] showed that PM limit of mGR does not exist. Indeed, the very (constraint) terms responsible for acausal mGR propagation [9] also imply the absence of a PM Bianchi identity and hence of any accompanying gauge symmetry [10].

At this point then, mGR is relegated at best to an effective theory of dubious physical relevance. On the other hand BMG, although at grave risk of suffering similar 
difficulties, might still conceivably be viable. In particular, since mGR solutions in a given background do not solve the BMG field equations, the mGR no-go results are not directly applicable [21]. As evidence for a PM BMG model, in [22] a vacuum solution admitting a PM linearization was exhibited. A healthy linearization being only the very first step for constructing consistent interactions, given the problems faced by the PM limits of $\mathrm{mGR}$ and $\mathrm{CG}$, one could reasonably infer their nonexistence in BMG. On the other hand, a PM limit of BMG would likely shield it from mGR's acausality difficulties because not only would a PM gauge invariance remove the offending helicity zero mode, but it would also imply absence of the fielddependent first derivative terms (that were responsible for mGR acausalities) in the scalar constraint of neighboring untuned (non-PM) BMG models. Nonexistence of a nonlinear PM gauge invariance of BMG is our subject.

\section{THE MODEL}

The dynamical fields are a pair of metric tensors $\left(g_{\mu \nu}, \bar{g}_{\mu \nu}\right)$; their action has a cosmological EinsteinHilbert term for each, plus a "mass term" $V$ depending algebraically on $(g, \bar{g})$ :

$$
\begin{aligned}
S= & M^{2} \int \sqrt{-g}(R(g)+2 \Lambda)+\mu^{4} \int V(g, \bar{g}) \\
& +\bar{M}^{2} \int \sqrt{-\bar{g}}(R(\bar{g})+2 \bar{\Lambda}) .
\end{aligned}
$$

The (mass-dimension) parameters $(M, \bar{M})$ and $\mu$ encode, respectively, the two Planck, and the FP, masses.

The equations of motion are

$$
\begin{aligned}
\mathcal{G}_{\mu \nu} & :=G_{\mu \nu}(g)-\Lambda g_{\mu \nu}-\frac{\mu^{4}}{M^{2}} \tau_{\mu \nu}=0 \\
& =G_{\mu \nu}(\bar{g})-\bar{\Lambda} \bar{g}_{\mu \nu}-\frac{\mu^{4}}{\bar{M}^{2}} \bar{\tau}_{\mu \nu}=: \bar{G}_{\mu \nu},
\end{aligned}
$$

where $\tau_{\mu \nu}$ and $\bar{\tau}_{\mu \nu}$ are, respectively (up to metric determinant factors), the $g$ and $\bar{g}$ variations of the mass term.

The analysis of preferred mass terms is greatly simplified by working in terms of a corresponding pair of vierbeins $\left(e_{\mu}{ }^{m}, f_{\mu}{ }^{m}\right)$, rather than metrics:

$$
g_{\mu \nu}=e_{\mu}{ }^{m} \eta_{m n} e_{\nu}{ }^{n}, \quad \bar{g}_{\mu \nu}=f_{\mu}{ }^{m} \eta_{m n} f_{\nu}{ }^{n} .
$$

Spin (as well as metric) connections are the Levi-Civita (Christoffel) ones: $\omega_{\mu}{ }^{m}{ }_{n}:=\omega_{\mu}{ }_{n}{ }_{n}(e)$ and $\bar{\omega}_{\mu}{ }^{m}{ }_{n}:=$ $\omega_{\mu}{ }_{n}(f)$. We fix half of the local $S O(3,1) \times S O(3,1)$ vierbein ambiguity by requiring

$$
\varphi_{\mu \nu}:=f_{\mu}{ }^{m} e_{\nu m}=f_{\nu}{ }^{m} e_{\mu m} .
$$

These six symmetry conditions are consistent with the field equations, but leave a further six redundancies encoded by the algebraic (Stückelberg) gauge symmetry

$$
e_{\mu}{ }^{m} \mapsto \Lambda_{n}^{m} e_{\mu}{ }^{n}, \quad f_{\mu}{ }^{m} \mapsto \Lambda_{n}^{m} f_{\mu}{ }^{n} .
$$

(This is the diagonal subgroup of the two local Lorentz symmetries.) The underlying d.o.f. count is unchanged, the $10+10=20$ metric components have been replaced by $16+16=32$ vierbein components subject to 6 conditions (2) and 6 algebraic gauge redundancies. Before analyzing the d.o.f. count, we specialize to the "preferred" mass term

$$
V \sim \epsilon_{m n r s} e^{m} \wedge e^{n} \wedge f^{r} \wedge f^{s} .
$$

The other pair of allowed couplings, $\epsilon_{\text {mnrs }} e^{m} \wedge f^{n} \wedge f^{r} \wedge f^{s}$ and $\epsilon_{\text {mnrs }} e^{m} \wedge e^{n} \wedge e^{r} \wedge f^{s}$, are not relevant for the putative PM limit suggested in [23]. The PM mGR studies of $[10,20]$ ruled these terms out there; this implies that they are very unlikely to be allowable PM couplings in BMG either.

For inverse vierbeins we employ $e^{\mu}{ }_{m}$ and $f^{\mu}{ }_{m}$ :

$$
e_{\mu}^{m} e_{n}^{\mu}=\delta_{n}^{m}=f_{\mu}{ }^{m} f_{n}^{\mu},
$$

while

$$
\left(\varphi_{\mu \nu}\right)^{-1}=e_{m}^{\mu} f_{m}^{\nu}=: \chi^{\mu \nu}=\chi^{\nu \mu} .
$$

In the absence of a metric tensor, vectors and covectors can no longer be identified, while in the presence of two metrics this identification is no longer unique. There are various situations where we do want to use solely one or the other metric (and its vierbeins) to manipulate indices. When these arise we will write $\stackrel{g}{=}$ or $\stackrel{\bar{g}}{=}$. For example, given a pair of covectors $v_{\mu}$ and $w_{\nu}$, we will write $X \stackrel{g}{=} v^{\mu} w_{\mu}$ to denote the equation $X=g^{\mu \nu} v_{\mu} w_{\nu}$ and $X \stackrel{\bar{g}}{=} v^{\mu} w_{\mu}$ for $X=$ $\bar{g}^{\mu \nu} v_{\mu} w_{\nu}$. In mixed situations, we will indicate explicitly how indices are handled.

\section{THE VECTOR CONSTRAINT AND BIANCHI IDENTITY}

For any choice of tensor density $V(g, \bar{g})$, the model has a single manifest dynamical diffeomorphism invariance, under which

$$
\begin{aligned}
& \delta g_{\mu \nu}=\mathcal{L}_{\xi} g_{\mu \nu}=\nabla_{\mu} \xi_{\nu}+\nabla_{\nu} \xi_{\mu}, \\
& \delta \bar{g}_{\mu \nu}=\mathcal{L}_{\xi} \bar{g}_{\mu \nu}=\bar{\nabla}_{\mu} \xi_{\nu}+\bar{\nabla}_{\nu} \xi_{\mu} .
\end{aligned}
$$

(Here $\nabla$ and $\bar{\nabla}$ are the Levi-Civita connections of $g$ and $\bar{g}$, respectively.) This local invariance immediately implies the vector Bianchi identity

$$
M^{2} \sqrt{-g} g^{\mu \nu} \nabla_{\mu} G_{\nu \rho}+\bar{M}^{2} \sqrt{-\bar{g}} \bar{g}^{\mu \nu} \bar{\nabla}_{\mu} \overline{\mathcal{G}}_{\nu \rho}=0 .
$$

It also reduces the (apparent) d.o.f. count to $10+10-2 \times$ $4=12$ in the usual way, but there are still further constraints.

For the particular choice of mass term (3), using (2), we have

$$
\tau_{\mu \nu} \stackrel{g}{=} 2\left(\varphi_{\mu \rho}-g_{\mu \rho} \varphi_{\sigma}^{\sigma}\right) \varphi_{\nu}^{\rho}+g_{\mu \nu}\left(\varphi_{\rho}^{\rho} \varphi_{\sigma}^{\sigma}-\varphi_{\rho}^{\sigma} \varphi_{\sigma}^{\rho}\right)
$$

and the same structure for $\bar{\tau}_{\mu \nu}$, with all quantities barred. Taking divergences, after some algebra (see [10]), we learn 


$$
\begin{aligned}
\frac{M^{2}}{\mu^{4}} \mathcal{C}_{\mu}:= & g_{\mu \nu} \chi^{\nu \rho} \nabla^{\sigma} G_{\rho \sigma} \\
& \stackrel{g}{=}-2 \varphi^{\nu \rho}\left(K_{\nu \rho \mu}-g_{\nu \rho} K_{\sigma}{ }^{\sigma}{ }_{\mu}+g_{\mu \nu} K_{\sigma}{ }^{\sigma}{ }_{\rho}\right)=0, \\
\frac{\bar{M}^{2}}{\mu^{4}} \overline{\mathcal{C}}_{\mu}:= & \bar{g}_{\mu \nu} \chi^{\nu \rho} \bar{\nabla}^{\sigma} \bar{G}_{\rho \sigma} \\
& \stackrel{\bar{g}}{=}+2 \varphi^{\nu \rho}\left(K_{\nu \rho \mu}-\bar{g}_{\nu \rho} K_{\sigma}{ }_{\mu}{ }_{\mu}+\bar{g}_{\mu \nu} K_{\sigma}{ }_{\rho}{ }_{\rho}\right)=0 .
\end{aligned}
$$

The contorsion $K$ appearing above is the difference of spin connections,

$$
K_{\mu}{ }_{n}{ }_{n}:=\omega_{\mu}{ }^{m}{ }_{n}(e)-\omega_{\mu}{ }^{m}(f) .
$$

It measures the failure of parallelograms of one metric to close with respect to the other. In particular $\nabla_{[\mu} f_{\nu]}^{m}=$ $K_{[\mu}{ }^{m}|n| f_{\nu]}{ }^{n}$ and $\bar{\nabla}_{[\mu} e_{\nu]}^{m}=-K_{[\mu}{ }^{m}|n|{ }_{\nu]}{ }^{n}$.

Although $\mathcal{C}_{\mu}$ and $\overline{\mathcal{C}}_{\mu}$ appear to furnish the theory with two separate vector constraints, they are not independent, thanks to the vector Bianchi identity (4). Thus, at this juncture, by virtue of the single vector constraint, the d.o.f. count sits at $8=10+10-2 \times 4-1 \times 4$. To arrive (covariantly) at the claimed generic $7=5+2$ modes of a massive + massless "graviton" pair, we need to uncover exactly one further, scalar, constraint. To this end, it will be instructive to first analyze linearized BMG.

\section{LINEARIZED BMG}

To linearize BMG, one first needs physically interesting vacua. Reference [22] (whose linear analysis we confirm here) proposed that these can be obtained by considering tuned metrics

$$
\bar{g}_{\mu \nu}=\gamma g_{\mu \nu}
$$

where the strictly positive constant $\gamma$ is fixed by the equations of motion. Then, taking $g$ and (therefore also) $\bar{g}$ to be Einstein metrics, we have

$$
G_{\mu \nu}(g)-\lambda g_{\mu \nu}=0=G_{\mu \nu}(\bar{g})-\lambda g_{\mu \nu},
$$

where the $\bar{g}$-cosmological constant is $\bar{\lambda}=\lambda / \gamma$. The equations of motion (1) then imply $(\Lambda-\lambda) g_{\mu \nu}+\frac{\mu^{4}}{M^{2}} \tau_{\mu \nu}=$ $0=(\gamma \bar{\Lambda}-\lambda) g_{\mu \nu}+\frac{\mu^{4}}{\bar{M}^{2}} \bar{\tau}_{\mu \nu}$. Since for this configuration, $\bar{\tau}_{\mu \nu}=\frac{1}{\gamma} \tau_{\mu \nu}=6 g_{\mu \nu}$, we learn

$$
M^{2}(\Lambda-\lambda)+6 \gamma \mu^{4}=0=\bar{M}^{2}(\gamma \bar{\Lambda}-\lambda)+6 \mu^{4} .
$$

Consequently we must require $\lambda=\Lambda+\frac{6 \gamma \mu^{4}}{M^{2}}$. Thus, away from the first of two critical cases (these will coalesce to a single one below), where $\mu^{4} \neq \frac{M^{2} \bar{\Lambda}}{6}$ we find

$$
\gamma=\frac{\Lambda-\frac{6 \mu^{4}}{\bar{M}^{2}}}{\bar{\Lambda}-\frac{6 \mu^{4}}{M^{2}}}
$$

while for $\mu^{4}=\frac{M^{2} \bar{\Lambda}}{6}$, the parameter $\gamma$ remains undetermined. The second critical case at $\mu^{4}=\frac{\bar{M}^{2} \Lambda}{6}$ appears because it yields an illegal $\gamma=0$ rescaling (this also follows by symmetry in the two metrics).
Having determined the parameter conditions for existence of the "diagonal" BMG Einstein solution, we now study its fluctuations. Linearizing

$$
g_{\mu \nu}=g_{\mu \nu}^{E}+h_{\mu \nu}, \quad \bar{g}_{\mu \nu}=\gamma g_{\mu \nu}^{E}+\bar{h}_{\mu \nu},
$$

we find $\varphi_{\mu \nu} \approx \sqrt{\gamma} g_{\mu \nu}^{E}+\frac{\sqrt{\gamma}}{2} h_{\mu \nu}+\frac{1}{2} \frac{1}{\sqrt{\gamma}} \bar{h}_{\mu \nu}$ and thus

$$
\begin{aligned}
& \tau_{\mu \nu} \approx 6 \gamma g_{\mu \nu}^{E}+8 \gamma\left(h_{\mu \nu}-\frac{1}{4} g_{\mu \nu}^{E} h\right)-2\left(\bar{h}_{\mu \nu}-g_{\mu \nu}^{E} \bar{h}\right), \\
& \bar{\tau}_{\mu \nu} \approx 6 g_{\mu \nu}^{E}-2\left(h_{\mu \nu}-g_{\mu \nu}^{E} h\right)+\frac{8}{\gamma}\left(\bar{h}_{\mu \nu}-\frac{1}{4} g_{\mu \nu}^{E} \bar{h}\right) .
\end{aligned}
$$

Here index manipulations and traces are performed with the Einstein background metric $g_{\mu \nu}^{E}$. Orchestrating the above, we obtain (in a matrix notation) the field equations,

$$
\begin{aligned}
& \left(\begin{array}{c}
G_{\mu \nu}^{L}(h)-\lambda h_{\mu \nu} \\
G_{\mu \nu}^{L}(\bar{h})-\lambda \bar{h}_{\mu \nu}
\end{array}\right) \\
& \quad=\frac{2 \mu^{4}}{M^{2} \bar{M}^{2}}\left(\begin{array}{cc}
\gamma \bar{M}^{2} & -M^{2} \\
-\gamma \bar{M}^{2} & M^{2}
\end{array}\right)\left(\begin{array}{c}
h_{\mu \nu}-g_{\mu \nu}^{E} h \\
\bar{h}_{\mu \nu}-g_{\mu \nu}^{E} \bar{h}
\end{array}\right) .
\end{aligned}
$$

The $2 \times 2$ "mass" matrix has eigenvalues 0 and $M^{2}+\gamma \bar{M}^{2}$. Thus, away from the disallowed values of $\mu$ given above, we find a (massless, massive) graviton pair. The linearized graviton mass parameter $m^{2}=4 \mu^{4}\left(\bar{M}^{-2}+\gamma M^{-2}\right)$ can still be changed arbitrarily by making further constant metric rescalings. For example, sending $g_{\mu \nu}^{E} \rightarrow \beta g_{\mu \nu}^{E}$ changes the $\left(m^{2}, \lambda\right)$ system to $\left(\beta m^{2}, \lambda\right)$. [The choice $\beta=\frac{1}{\sqrt{\gamma}}$ gives, for example, a mass that is symmetric in barred and unbarred quantities.] Precisely at the critical cases, this freedom drops out: consider the critical point, $\mu^{4}=\frac{M^{2} \bar{\Lambda}}{6}$. Although $\gamma$ is undetermined, we are still forced by Eq. (6) to set

$$
M^{2} \bar{\Lambda}=\bar{M}^{2} \Lambda,
$$

which yields equivalence of the two critical $\mu$ values. The cosmological constant of the background is now

$$
\lambda=\Lambda+\gamma \bar{\Lambda} .
$$

For the linearized equations of motion we find

$$
\left(\begin{array}{c}
G_{\mu \nu}^{L}(h)-\lambda h_{\mu \nu} \\
G_{\mu \nu}^{L}(\bar{h})-\lambda \bar{h}_{\mu \nu}
\end{array}\right)=\frac{1}{3}\left(\begin{array}{cc}
\gamma \bar{\Lambda} & -\Lambda \\
-\gamma \bar{\Lambda} & \Lambda
\end{array}\right)\left(\begin{array}{c}
h_{\mu \nu}-g_{\mu \nu}^{E} h \\
\bar{h}_{\mu \nu}-g_{\mu \nu}^{E} \bar{h}
\end{array}\right),
$$

which describes a massless graviton as well as a mode with mass

$$
m^{2}=\frac{2 \lambda}{3}
$$

This is precisely the partially massless tuning and fuels hope that the full BMG may enjoy an (interacting) partially massless limit [23].

Diagonalizing the field equations, we find that $h_{\mu \nu}^{\text {grav }}:=h_{\mu \nu}+\bar{h}_{\mu \nu}$ describes the linearized, cosmological, graviton excitation, $G_{\mu \nu}^{L}\left(h^{\text {grav }}\right)-\lambda h_{\mu \nu}^{\text {grav }}=0$, while $h_{\mu \nu}^{\mathrm{PM}}:=\gamma \bar{\Lambda} h_{\mu \nu}-\Lambda \bar{h}_{\mu \nu}$ is the PM mode which obeys 


$$
G_{\mu \nu}^{\mathrm{PM}}:=G_{\mu \nu}^{L}\left(h^{\mathrm{PM}}\right)-\frac{4 \lambda}{3} \tilde{h}_{\mu \nu}^{\mathrm{PM}}=0,
$$

(where $\tilde{h}_{\mu \nu}:=h_{\mu \nu}-\frac{1}{4} g_{\mu \nu}^{E} h$ ). In particular, the linear Bianchi identity is

$$
\left(\nabla^{\mu} \nabla^{\nu}+\frac{\lambda}{3} g_{E}^{\mu \nu}\right) G_{\mu \nu}^{L}\left(h^{\mathrm{PM}}\right) \equiv 0,
$$

and corresponds to the PM invariance $\delta h_{\mu \nu}=\left(\nabla_{\mu} \nabla_{\nu}+\right.$ $\left.\frac{\lambda}{3} g_{\mu \nu}^{E}\right) \alpha(x)$. As a forewarning, we note that the combination of linearized fluctuations in $h_{\mu \nu}^{\mathrm{PM}}$ depends on the undetermined parameter $\gamma$, which will limit the usefulness of the linear analysis when searching for a nonlinear extension.

\section{NO PM BMG?}

Our main argument against PM BMG is that a Bianchi identity and associated nonlinear gauge invariance (at some critical point), extending the linear one exhibited above, is unlikely to exist. One problem is that no covariant formulation of the BMG scalar constraint responsible for the $7=2+5$ d.o.f. count of an interacting graviton and massive spin 2 excitation has yet been found: to date all groups claiming that the sixth, massive ghostlike excitation is absent for distinguished BMG mass terms relied on a $3+1$ ADM-type analysis [24]. Strangely enough, although these analyses claim to prove that BMG propagates 7 d.o.f.'s, none of them are able to show that a PM parameter-tuning yields a 6 d.o.f. PM limit, even though for cosmological PF models one sees this quite directly (in fact, in a $3+1$ split, the zero helicity part of the action becomes exactly zero at the PM point [25]). On the one hand, we suspect that an exhaustive PM BMG no-go proof will require a computation of the Poisson bracket algebra of the (noncovariant) constraints in order to check for an enhancement of the first class symmetry algebra. If there really is no covariant formulation of the scalar constraint, however [26], then it is already very difficult to believe that a covariant gauge symmetry arises - recall that in the free theory, the partially massless gauge symmetry corresponds to a double derivative Bianchi identity, obtained from a masscosmological constant tuning limit of the double divergence constraint [27].

To see why mGR enjoys a covariant system of constraints, while BMG seems not to, consider a putative nonlinear Bianchi identity of the form

$$
\nabla^{?} \nabla^{?} \cdot \mathcal{G}-\bar{\nabla} ? \bar{\nabla}^{?} \cdot \bar{G}+\text { l.o.t. } \equiv 0,
$$

where the lower order terms (l.o.t.) involve fewer derivatives on the field equations $G$ and $\overline{\mathcal{G}}$. On general grounds, this identity can involve no more than two covariant derivatives. We have denoted the contraction of these on the field equations by $?$ to indicate that the tensor contraction of indices here is still to be determined. We can study the identical vanishing of this quantity order by order in derivatives on the two dynamical fields $g_{\mu \nu}$ and $\bar{g}_{\mu \nu}$. In particular we can first focus on $g_{\mu \nu}$. This allows us to steal results from the known mGR case. There, one knows that the contraction of the first covariant derivative is the one giving the vector constraint/Bianchi identity, namely $g^{\mu \rho} \nabla_{\mu} G_{\rho \nu}$. In other words, the Bianchi identity should follow from an appropriate divergence $\nabla ? \mathcal{C}$ of the vector constraint (recall that $\mathcal{C}_{\mu}$ and $\overline{\mathcal{C}}_{\mu}$ are not independent)

$$
\mathcal{C}_{\mu} \stackrel{g}{=}-\frac{2 \mu^{4}}{M^{4}} \varphi^{\nu \rho}\left(K_{\nu \rho \mu}-g_{\nu \rho} K_{\sigma}{ }^{\sigma}{ }_{\mu}+g_{\mu \nu} K_{\sigma}{ }^{\sigma}\right) .
$$

Again, by working order by order in derivatives on the independent field $g_{\mu \nu}$ we deduce from the mGR case that any putative Bianchi identity follows from the particular choice of divergence $g^{\mu \nu} \nabla_{\mu} \mathcal{C}_{\nu}$. This quantity has been computed in [10], but here we need only focus on terms involving two derivatives on the dynamical variables which can be computed using the following identity relating Riemann tensors of differing connections to the contorsion:

$$
R_{\mu \nu}{ }^{m}{ }_{n}(\omega)-R_{\mu \nu}{ }^{m}(\bar{\omega})=2\left(\nabla_{[\mu} K_{\nu]}{ }_{n}{ }_{n}-K_{[\mu}{ }^{m}|r|{ }_{\nu]}{ }^{r}{ }^{n}\right) \text {. }
$$

Using this, we find at leading derivative order

$$
\begin{aligned}
g^{\mu \nu} \nabla_{\mu} \mathcal{C}_{\nu} \stackrel{g}{=} & -\frac{2 \mu^{4}}{M^{4}}\left(\varphi^{\nu \rho}\left[R(\omega)_{\mu \nu \rho}{ }^{\mu}-R(\bar{\omega})_{\mu \nu \rho}{ }^{\mu}\right]\right. \\
& \left.+\frac{1}{2} \varphi_{\rho}^{\rho}\left[R(\omega)_{\mu \nu}{ }^{\nu \mu}-R(\bar{\omega})_{\mu \nu}{ }^{\nu \mu}\right]\right)+ \text { l.o.t. }
\end{aligned}
$$

There are various lessons to be learned from this relation: first, for mGR where $R(\bar{\omega})_{\mu \nu}{ }_{n}{ }_{n}$ is the Riemann tensor of the fiducial background, the existence of the scalar constraint is immediate, because the Riemann tensor for $g_{\mu \nu}$ appears only in its Ricci or Ricci-scalar form, both of which can be canceled at leading derivative order by adding a trace of the field equation $\mathcal{G}_{\mu \nu}$ [28]. If one further takes the background to be constant curvature, the existence of a PM mGR limit hinges on the lower order terms. These were shown not to cancel in [10], thus ruling out the PM mGR theory. If we were to require that the PM mGR limit also held for Einstein backgrounds (after all, these are sufficient for consistent linear PM propagation $[16,25]$ ), the terms $R(\bar{\omega})$ already destroy that model. They are, in fact, also lethal for the prospects of a PM BMG. The main point is simple: the Riemann tensor of the second metric $\bar{g}_{\mu \nu}$ does not appear as a Ricci or Ricci scalar and thus cannot be removed using the field equation $\bar{G}_{\mu \nu}$. Note that this does not contradict previous claims that the sixth massive ghost mode is absent, because the leading double time derivative terms can be removed by choosing a time slicing adapted to the metric $g_{\mu \nu}$ [29].

We have experimented with other possible contractions $\nabla^{?} \mathcal{C}$, as well as using identities for the contorsion following from the relation (2) and found no way to avoid the appearance of "bare" Riemann tensors in the putative Bianchi identity. These cannot be converted to Ricci tensor or scalars nor in turn traded for equations of motion. By way of warning, even supposing that a combination of double divergences and traces of field equations canceling all second derivatives of $g$ and $\bar{g}$ existed, a Bianchi identity further requires all remaining terms to cancel off shell. This failed 
spectacularly for the mGR model. Moreover, the coupling of two metrics produces a new covariant tensor-the contorsion-and there is no mechanism (aside from conformal symmetry which yields relatively ghost CG) preventing this from happening again. (However, unlike our previous study of PM mGR [10], we have not conducted an order by order expansion in fields and derivatives.)

Aside from the linear analysis of [22] that we summarized in Sec. IV, the other piece of purported PM BMG evidence is that a certain truncation of a higher derivative "version" of (tuned) BMG yields CG. By virtue of its local conformal and diffeomorphism invariances, CG (nonlinearly) propagates PM and graviton, relative-ghost modes $[18,19]$. However, the higher derivative model studied in [22] is not equivalent to BMG because it is obtained by the generally illegitimate procedure of plugging dynamical field equations back into the action principle [30]: consider a simple two-field model $S[\phi, \psi]=\int\left(\frac{1}{2} \phi \square \phi+\frac{1}{2} \psi \square \psi-\phi \psi\right)$ (in obvious mass units); the equations of motion imply $\square \phi=\psi$ and $\square \psi=\phi$. Thus one might be tempted to conclude (by using $\psi=\psi(\phi):=\square \phi)$ that an equivalent model is $S[\phi]=$ $S[\phi, \psi(\phi)]=-\int \frac{1}{2} \phi\left[\square-\square^{3}\right] \phi$ with equations of motion $[\square-1][\square+1] \square \phi=0$. However this latter model has a solution $\square \phi=0$ that does not solve the original system of equations. That the same inconsistency afflicts the higher derivative model obtained from the bimetric theory is not hard to see. For the bimetric theory this "half-shell" procedure yields CG with higher order curvature corrections: $S=\int W^{2}+\mathcal{O}\left(R^{3}\right)$ (where $W$ denotes the Weyl tensor). So any conformally Einstein manifold is a leading order solution, in particular $G_{\mu \nu}(g)=\Lambda g_{\mu \nu}$. The bimetric equations of motion have the form $G_{\mu \nu}(g)-$ $\Lambda g_{\mu \nu}=\frac{\mu^{4}}{M^{2}} \tau_{\mu \nu}(g, \bar{g})$ plus the same with bars. Clearly we are now forced into the same corner, namely $\bar{g}_{\mu \nu}=0$ and in turn [31] $g_{\mu \nu}=0$. The fact that a certain truncation of a higher derivative theory-that itself is explicitly inequivalent to BMG-has a scalar gauge invariance does not imply a scalar gauge invariance of PM BMG.

Finally, even supposing we were to employ the higher derivative model as inspiration for a possible PM BMG gauge principle, since its action starts with that of
$\mathrm{CG}$, a putative PM gauge transformation would then begin with a Weyl transformation. Higher derivative corrections to these transformations can then be ignored by taking the special case where the gauge parameter is constant. However, this would imply existence of a rescaling symmetry, which is clearly incompatible with the higher order terms' dependence on powers of the dimensionful cosmological constant. (One might hope to modify this scaling symmetry by some sort of curvature-dependent extension reducing to unity in a low curvature limit, but this seems a pointless exercise given our previous no-go arguments.) We therefore conclude, with some confidence, that GR is indeed an isolated theory, like its non-Abelian Yang-Mills cousin.

\section{CONCLUSIONS}

We have argued that the existence of a PM version of BMG is crucial to its consistency. In particular a consistent PM could shield the model from causal inconsistencies of the type uncovered for mGR in $[9,10]$. We have further argued that the known no-go results for PM mGR make survival of a PM symmetry in BMG most unlikely. Supposing, as we believe, that this can be made rigorous, what are the prospects for improving BMG? Perhaps some string-inspired approach such as that of [33] (which attempts to restore consistency of massive charged $s=3 / 2,2$ interactions by embedding them in a consistent underlying framework) may help. (First steps in this direction have been made in [34], although even there instabilities are encountered.) This would likely entail also modifying the kinetic, Einstein curvature term (as recently attempted in [35]). Maintaining even the BMG vector constraint is difficult in such an approach, so the obstacles here seem high [36].

\section{ACKNOWLEDGMENTS}

A. W. thanks G. Gabadadze, F. Hassan, K. Hinterbichler and M. S. and A. W. thank N. Kaloper for discussions. S. D. was supported in part by NSF Grant No.PHY-1266107 and DOE Grant No. DE-FG02-164 92ER40701.
[1] A. Salam and J. Strathdee, Phys. Rev. 184, 1750 (1969); C. J. Isham, A. Salam, and J. Strathdee, Phys. Lett. 31B, 300 (1970); See also N. Rosen, Ann. Phys. (N.Y.) 84, 455 (1974); S. Deser, in Developments in GR (IOP, Bristol, 1990).

[2] B. Zumino, in Brandeis Univ. Lectures on Elementary Particles and Quantum Field Theory, edited by S. Deser, M. Grisaru, and H. Pendleton (MIT Press, Cambridge, MA, 1970), Vol. 2, p. 437.

[3] S. Deser and D. G. Boulware, Phys. Rev. D 6, 3368 (1972); Phys. Lett. 40B, 227 (1972).
[4] C. de Rham, G. Gabadadze, and A.J. Tolley, Phys. Rev. Lett. 106, 231101 (2011); Phys. Lett. B 711, 190 (2012); C. de Rham and G. Gabadadze, Phys. Rev. D 82, 044020 (2010); S. F. Hassan and R. A. Rosen, Phys. Rev. Lett. 108, 041101 (2012).

[5] S. F. Hassan and R. A. Rosen, J. High Energy Phys. 02 (2012) 126.

[6] K. Hinterbichler, Rev. Mod. Phys. 84, 671 (2012).

[7] A. Gruzinov, arXiv:1106.3972.

[8] C. Burrage, C. de Rham, L. Heisenberg, and A. J. Tolley, J. Cosmol. Astropart. Phys. 07 (2012) 004; P. de Fromont, 
C. de Rham, L. Heisenberg, and A. Matas, J. High Energy Phys. 07 (2013) 067.

[9] S. Deser and A. Waldron, Phys. Rev. Lett. 110, 111101 (2013); K. Izumi and Y.C. Ong, Classical Quantum Gravity 30, 184008 (2013); S. Deser, K. Izumi, Y.C. Ong, and A. Waldron, Phys. Lett. B 726, 544 (2013).

[10] S. Deser, M. Sandora, and A. Waldron, Phys. Rev. D 87, 101501 (2013).

[11] K. Johnson and E. C. G. Sudarshan, Ann. Phys. (N.Y.) 13, 126 (1961).

[12] G. Velo and D. Zwanziger, Phys. Rev. 186, 1337 (1969); See also S. Deser, V. Pascalutsa, and A. Waldron, Phys. Rev. D 62, 105031 (2000).

[13] S. Deser and A. Waldron, Nucl. Phys. B631, 369 (2002).

[14] For technical reasons only, these latest no-go results leave open one class of mass terms (cubic in the background metric); they most likely suffer from the same propagating tachyons.

[15] A. De Felice, A. E. Gumrukcuoglu, and S. Mukohyama, Phys. Rev. Lett. 109, 171101 (2012); A. De Felice, A.E. Gumrukcuoglu, C. Lin, and S. Mukohyama, J. Cosmol. Astropart. Phys. 05 (2013) 035; Classical Quantum Gravity 30, 184004 (2013).

[16] S. Deser and R. I. Nepomechie, Ann. Phys. (N.Y.) 154, 396 (1984); Phys. Lett. 132B, 321 (1983); A. Higuchi, Nucl. Phys. B282, 397 (1987); B325, 745 (1989); J. Math. Phys. (N.Y.) 28, 1553 (1987); 43, 6385(E) (2002); S. Deser and A. Waldron, Phys. Rev. Lett. 87, 031601 (2001); Nucl. Phys. B607, 577 (2001); Phys. Lett. B 513, 137 (2001); 513, 137 (2001); Nucl. Phys. B662, 379 (2003); Phys. Lett. B 603, 30 (2004); Phys. Rev. D 74, 084036 (2006); 87, 087702 (2013).

[17] Y. M. Zinoviev, Nucl. Phys. B770, 83 (2007); B821, 431 (2009).

[18] J. Maldacena, arXiv:1105.5632.

[19] S. Deser, E. Joung, and A. Waldron, Phys. Rev. D 86, 104004 (2012); J. Phys. A 46, 214019 (2013).

[20] C. de Rham, K. Hinterbichler, R. A. Rosen, and A.J. Tolley, Phys. Rev. D 88, 024003 (2013).

[21] The PM mGR no-go does restrict possible PM gauge invariances of the higher derivative model proposed in [22] in which, by solving algebraically for one metric in terms of the other, BMG is converted to a single metric model involving an infinite tower of higher derivative interactions. The authors of [22] claim this theory could constitute a renormalizable ghost-free model and, even more optimistically, that a PM gauge symmetry could protect the structure of these against deadly radiative corrections. We exhibit the pitfalls facing these claims in Sec. V.

[22] S.F. Hassan, A. Schmidt-May, and M. von Strauss, arXiv:1303.6940.

[23] S.F. Hassan, A. Schmidt-May, and M. von Strauss, arXiv:1208.1797; see also Y. Akrami, T.S. Koivisto, D. F. Mota, and M. Sandstad, arXiv:1306.0004.

[24] S. F. Hassan and R. A. Rosen, J. High Energy Phys. 04 (2012) 123; K. Nomura and J. Soda, Phys. Rev. D 86, 084052 (2012); J. Kluson, arXiv:1301.3296; arXiv:1303.1652; V. O. Soloviev and M. V. Tchichikina, arXiv:1211.6530; arXiv:1302.5096; D. Comelli, F. Nesti, and L. Pilo, arXiv:1305.0236; S. Alexandrov, K. Krasnov, and S. Speziale, J. High Energy Phys. 06 (2013) 068.

[25] S. Deser and A. Waldron, Phys. Lett. B 508, 347 (2001).
[26] Clever use of auxiliary fields might perhaps permit lifting the constraints to covariant form, but that need not necessarily be useful in itself.

[27] Here, we are assuming that any putative PM gauge invariance (and thus Bianchi identity) is covariant with respect to diffeomorphisms. One might in principle envision a situation where the commutator of diffeomorphisms and PM gauge symmetries vanishes on shell, thus allowing a noncovariant PM gauge invariance without introducing new first class symmetries. We strongly doubt that any such noncovariant PM gauge invariance exists, however.

[28] C. Deffayet, J. Mourad, and G. Zahariade, J. Cosmol. Astropart. Phys. 01 (2013) 032.

[29] We are not claiming that the constraint analysis relies on this choice, only that obtaining the scalar constraint from the above combination does.

[30] This point is actually conceded in an appendix of [22], but there it is suggested-based on the example of a Gaussian path integral of a two-scalar, free theory - that by carefully keeping track of sources, ghosts could be somehow removed. In any case, it is inconsistent to perform interacting path integrals by going (even partially) on shell, that is by replacing fields by solutions to their field equations.

[31] In fact, PM invariance of the higher derivative model is intimately tied to the PM mGR Bianchi identity, as can be seen by applying the 1.5 order formalism (see [32]): the higher derivative model is obtained by solving for one metric $\bar{g}$ (say) in terms of the other by its algebraic field equation, $\frac{\delta S(g, \bar{g})}{\delta g}=0 \Rightarrow \bar{g}=\bar{g}(g)$. Inserting this relation back in the action gives a higher derivative model $S(g):=$ $S(g, \bar{g}(g)$ ) (whose leading term is claimed to be the CG action [22]) and can then easily be varied:

$$
\begin{aligned}
\delta S(g) & =\frac{\delta S(g, \bar{g}(g))}{\delta g} \delta g+\left.\frac{\delta S(g, \bar{g})}{\delta \bar{g}}\right|_{\bar{g}=\bar{g}(g)} \frac{\delta \bar{g}(g)}{\delta g} \delta g \\
& =\left.\frac{\delta S(g, \bar{g})}{\delta \bar{g}}\right|_{\bar{g}=\bar{g}(g)} \frac{\delta \bar{g}(g)}{\delta g} \delta g .
\end{aligned}
$$

The $g$ variation vanishes thanks to the 1.5 order method [because $\bar{g}$ obeys the field equations $\frac{\delta S(g, \bar{g})}{\delta g}=0$ ]. Moreover, $\frac{\delta S(g, \bar{g})}{\delta \bar{g}}=0$ is exactly the equation of motion of mGR for $\bar{g}$. The functional relation $\frac{\delta S(g, \bar{g})}{\delta \bar{g}} \delta \bar{g}=0$ (for general $\bar{g}$ ) amounts precisely to a PM ${ }^{\delta g}$ GR Bianchi identity. Its absence has by now been independently established by three groups [10,17,20] [although conceivably, the PM mGR Bianchi identity failure could vanish on the subspace $\bar{g}=\bar{g}(g)]$.

[32] P. Van Nieuwenhuizen, Phys. Rep. 68, 189 (1981).

[33] M. Porrati, R. Rahman, and A. Sagnotti, Nucl. Phys. B846, 250 (2011); M. Porrati and R. Rahman, Phys. Rev. D 80, 025009 (2009); 84, 045013 (2011).

[34] E. Kiritsis, J. High Energy Phys. 11 (2006) 049; O. Aharony, A. B. Clark, and A. Karch, Phys. Rev. D 74, 086006 (2006); E. Kiritsis and V. Niarchos, J. High Energy Phys. 08 (2008) 044; Nucl. Phys. B812, 488 (2009).

[35] K. Hinterbichler, arXiv:1305.7227; See also S. Folkerts, A. Pritzel, and N. Wintergerst, arXiv:1107.3157.

[36] Since our work's posting, absence of a PM version of BMG was independently confirmed by M. Fasiello and A. J. Tolley, arXiv:1308.1647. 